\title{
Can nutritional interventions change major clinical outcomes?
}

\author{
Sho Horikita ${ }^{1}$, Masamitsu Sanui ${ }^{i^{*}}$ (D) and Alan K. Lefor ${ }^{2}$
}

Keywords: Enteral nutrition, Parenteral nutrition, Critically ill, Permissive underfeeding

In the NUTRIREA-2 trial [1] recently published in the Lancet, the authors assessed whether early enteral nutrition (EN) decreases mortality compared with early parenteral nutrition $(\mathrm{PN})$ in patients undergoing invasive mechanical ventilation and vasopressor support for shock, with normo-caloric targets for both routes of support. As the primary endpoint, mortality on day 28 was comparable between the groups. However, we believe that nutritional protocols for both study groups deviated from current standards of nutritional therapy for critically ill patients, which makes interpretation of the results difficult.

There are no clear data showing that EN targeting full caloric requirements immediately after admission is superior to permissive hypocaloric EN [2, 3]. Recent large randomized controlled trials (RCTs) even suggest that full caloric EN has some disadvantages over hypocaloric $\mathrm{EN}$, including gastrointestinal intolerance [2]. The latest Society of Critical Care Medicine and American Society for Parenteral and Enteral Nutrition guidelines [4] suggest that EN is the preferred nutritional route for the majority of critically ill patients. Therefore, there is no clear justification for patients in refractory shock to receive full-caloric EN as in the NUTRIREA-2 trial [1], although these unstable patients may tolerate trophic feeding or underfeeding. In the real world, critical care staff would take significant advantage of less gastrointestinal complications along with non-inferiority of hypocaloric to normocaloric EN.

Also, there are no definite data showing that early PN beginning within a few days of ICU admission, either supplemental or exclusive PN to meet full-caloric goals,

\footnotetext{
* Correspondence: msanui@mac.com

${ }^{1}$ Department of Anesthesiology and Critical Care Medicine, Jichi Medical University Saitama Medical Center, 1-847 Amanumacho Omiya, Saitama, Saitama 330-8503, Japan

Full list of author information is available at the end of the article
}

has a significant clinical benefit. In the EPaNIC trial [5], early PN supplementation to hypocaloric EN for full caloric targets did not improve mortality in critically ill patients, but did increase the rate of infections. Therefore, full-caloric PN is not the current standard nutritional therapy for critically ill patients.

For unstable patients requiring vasopressors and mechanical ventilation, we believe that trophic EN is the first-line nutritional therapy. Based on the fact that none of the EN or PN nutritional protocols $[1-3,5]$ showed a benefit of nutritional therapy on clinically important outcomes in critically ill patients, additional efforts to investigate a particular nutritional therapy to improve clinical outcomes during the acute phase may not be advocated. It is rather reasonable to investigate a nutritional therapy to do the least harm. We need to appreciate the idea that nutrition does not give strength to patients, but can potentially do harm to patients.

\section{Abbreviations \\ EN: Enteral nutrition; PN: Parenteral nutrition; RCT: Randomized controlled trial}

\section{Authors' contributions}

$\mathrm{SH}$ and MS were involved in conception and writing of the manuscript. AKL was involved in critical revision of the manuscript. All authors read and approved the final version of the manuscript.

\section{Competing interests}

The authors declare that they have no competing interests.

\section{Publisher's Note}

Springer Nature remains neutral with regard to jurisdictional claims in published maps and institutional affiliations.

\section{Author details}

'Department of Anesthesiology and Critical Care Medicine, Jichi Medical University Saitama Medical Center, 1-847 Amanumacho Omiya, Saitama, Saitama 330-8503, Japan. ${ }^{2}$ Department of Surgery, Jichi Medical University, 3311-1 Yakushiji, Shimotsuke-shi, Tochigi 329-0498, Japan. 
Received: 17 May 2018 Accepted: 24 May 2018

Published online: 08 June 2018

\section{References}

1. Reignier J, Boisramé-Helms J, Brisard L, et al. Enteral versus parenteral early nutrition in ventilated adults with shock: a randomised, controlled,

multicentre, open-label, parallel-group study (NUTRIREA-2). Lancet. 2018;391:

133-43.

2. National Heart, Lung, and Blood Institute Acute Respiratory Distress Syndrome (ARDS) Clinical Trials Network, Rice TW, Wheeler AP, Thompson $B T$, et al. Initial trophic vs full enteral feeding in patients with acute lung injury: the EDEN randomized trial. JAMA. 2012;307:795-803.

3. Arabi YM, Aldawood AS, Haddad $\mathrm{SH}$, et al. Permissive underfeeding or standard enteral feeding in critically ill adults. N Engl J Med. 2015;372:2398-408.

4. Taylor BE, McClave SA, Martindale RG, et al. Guidelines for the provision and assessment of nutrition support therapy in the adult critically ill patient: Society of Critical Care Medicine (SCCM) and American Society for Parenteral and Enteral Nutrition (A.S.P.E.N.). Crit Care Med. 2016;44:390-438.

5. Casaer MP, Mesotten D, Hermans G, et al. Early versus late parenteral nutrition in critically ill adults. N Engl J Med. 2011;365:506-17. 